University of Wollongong

Research Online

Faculty of Engineering and Information

Faculty of Engineering and Information

Sciences - Papers: Part A

Sciences

$1-1-2015$

\title{
The evaluation of a 2D diode array in "magic phantom" for use in high dose rate brachytherapy pretreatment quality assurance
}

\author{
Anthony A. Espinoza \\ University of Wollongong, aae718@uowmail.edu.au \\ Marco Petasecca \\ University of Wollongong, marcop@uow.edu.au \\ Iolanda Fuduli \\ University of Wollongong, if473@uowmail.edu.au \\ Andrew Howie \\ St George Hospital \\ Joseph A. Bucci \\ St George Hospital
}

See next page for additional authors

Follow this and additional works at: https://ro.uow.edu.au/eispapers

Part of the Engineering Commons, and the Science and Technology Studies Commons

Research Online is the open access institutional repository for the University of Wollongong. For further information contact the UOW Library: research-pubs@uow.edu.au 


\title{
The evaluation of a $2 \mathrm{D}$ diode array in "magic phantom" for use in high dose rate brachytherapy pretreatment quality assurance
}

\begin{abstract}
Purpose: High dose rate (HDR) brachytherapy is a treatment method that is used increasingly worldwide. The development of a sound quality assurance program for the verification of treatment deliveries can be challenging due to the high source activity utilized and the need for precise measurements of dwell positions and times. This paper describes the application of a novel phantom, based on a 2D $11 \times 11$ diode array detection system, named "magic phantom" (MPh), to accurately measure plan dwell positions and times, compare them directly to the treatment plan, determine errors in treatment delivery, and calculate absorbed dose. Methods: The magic phantom system was CT scanned and a 20 catheter plan was generated to simulate a nonspecific treatment scenario. This plan was delivered to the MPh and, using a custom developed software suite, the dwell positions and times were measured and compared to the plan. The original plan was also modified, with changes not disclosed to the primary authors, and measured again using the device and software to determine the modifications. A new metric, the "position-time gamma index," was developed to quantify the quality of a treatment delivery when compared to the treatment plan. The MPh was evaluated to determine the minimum measurable dwell time and step size. The incorporation of the TG-43U1 formalism directly into the software allows for dose calculations to be made based on the measured plan. The estimated dose distributions calculated by the software were compared to the treatment plan and to calibrated EBT3 film, using the 2D gamma analysis method. Results: For the original plan, the magic phantom system was capable of measuring all dwell points and dwell times and the majority were found to be within $0.93 \mathrm{~mm}$ and $0.25 \mathrm{~s}$, respectively, from the plan. By measuring the altered plan and comparing it to the unmodified treatment plan, the use of the position-time gamma index showed that all modifications made could be readily detected. The MPh was able to measure dwell times down to $0.067 \pm 0.001 \mathrm{~s}$ and planned dwell positions separated by $1 \mathrm{~mm}$. The dose calculation carried out by the MPh software was found to be in agreement with values calculated by the treatment planning system within $0.75 \%$. Using the $2 \mathrm{D}$ gamma index, the dose map of the MPh plane and measured EBT3 were found to have a pass rate of over $95 \%$ when compared to the original plan. Conclusions: The application of this magic phantom quality assurance system to HDR brachytherapy has demonstrated promising ability to perform the verification of treatment plans, based upon the measured dwell positions and times. The introduction of the quantitative position-time gamma index allows for direct comparison of measured parameters against the plan and could be used prior to patient treatment to ensure accurate delivery.
\end{abstract}

\section{Keywords}

brachytherapy, quality assurance, magic plate, gamma index, diode array

Disciplines

Engineering | Science and Technology Studies

\section{Publication Details}

Espinoza, A., Petasecca, M., Fuduli, I., Howie, A., Bucci, J., Corde, S., Jackson, M., Lerch, M. L. F. \& Rosenfeld, A. B. (2015). The evaluation of a 2D diode array in "magic phantom" for use in high dose rate brachytherapy pretreatment quality assurance. Medical Physics, 42 (2), 663-673.

\section{Authors}

Anthony A. Espinoza, Marco Petasecca, Iolanda Fuduli, Andrew Howie, Joseph A. Bucci, Stephanie Corde, Michael Jackson, Michael L. F Lerch, and Anatoly B. Rosenfeld

This journal article is available at Research Online: https://ro.uow.edu.au/eispapers/3328 


\section{The evaluation of a two dimensional diode array in "magic phantom" for use in high dose rate brachytherapy pre-treatment quality assurance}

A. Espinoza, M. Petasecca and I. Fuduli

Centre for Medical Radiation Physics, University of Wollongong, New South Wales 2522, Australia

A. Howie and J. Bucci

St George Hospital Cancer Care Centre, New South Wales 2217, Australia

S. Corde and M. Jackson

Department of Radiation Oncology, Prince of Wales Hospital, New South Wales 2031, Australia

M.L.F. Lerch and A.B. Rosenfeld

Centre for Medical Radiation Physics, University of Wollongong, New South Wales 2522, Australia

Purpose: High dose rate (HDR) brachytherapy is a treatment method that is used increasingly worldwide. The development of a sound quality assurance (QA) program for the verification of treatment deliveries can be challenging due to the high source activity utilized and the need for precise measurements of dwell positions and times. This paper describes the application of a novel phantom, based on a two dimensional 11x11 diode array detection system, named "magic phantom", to accurately measure plan dwell positions and times, compare them directly to the treatment plan, determine errors in treatment delivery and calculate absorbed dose.

Methods: The "magic phantom" (MPh) system was CT scanned and a 20 catheter plan was generated to simulate a nonspecific treatment scenario. This plan was delivered to the MPh and, using a custom developed software suite, the dwell positions and times were measured and compared to the plan. The original plan was also modified, with changes not disclosed to the primary authors, and measured again using the device and software to determine the modifications. A new metric, the "position-time gamma index", was developed to quantify the quality of a treatment delivery when compared to the treatment plan. The MPh was evaluated to determine the minimum measurable dwell time and step size. The incorporation of the TG-43U1 formalism directly into the software allows for dose calculations to be made based on the measured plan. The estimated dose distributions calculated by 
the software were compared to the treatment plan and to calibrated EBT3 film, using the 2D gamma analysis method.

Results: For the original plan, the "magic phantom" system was capable of measuring all dwell points and dwell times and the majority were found to be within $0.93 \mathrm{~mm}$ and $0.25 \mathrm{~s}$, respectively, from the plan. By measuring the altered plan and comparing it to the unmodified treatment plan, the use of the position-time gamma index showed that all modifications made could be readily detected. The MPh was able to measure dwell times down to $0.067 \pm 0.001 \mathrm{~s}$ and planned dwell positions separated by 1 $\mathrm{mm}$. The dose calculation carried out by the MPh software was found to be in agreement with values calculated by the treatment planning system within $0.75 \%$. Using the 2D gamma index, the dose map of the MPh plane and measured EBT3 were found to have a pass rate of over $95 \%$ when compared to the original plan.

Conclusions: Our application of the "magic phantom" quality assurance system to HDR brachytherapy has demonstrated promising ability to perform the verification of treatment plans, based upon the measured dwell positions and times. The introduction of the quantitative position-time gamma index allows for direct comparison of measured parameters against the plan, and could be used prior to patient treatment to ensure accurate delivery.

\section{Introduction}

Due to the severe consequences associated with the incorrect delivery of High Dose Rate (HDR) brachytherapy treatments, it is essential that the measurement of source dwell times and verification of dwell location can be undertaken with high precision. Human and calculation errors introduced during the treatment planning and preparation processes, in addition to incorrect calibration of the HDR afterloader, can potentially lead to incorrect dwell location and timing during treatment, resulting in the misadministration of dose to the treatment volume $\mathrm{e}^{1,2,3}$.

Brachytherapy treatment planning systems (TPS) employ algorithms based on the AAPM TG-43U1 protocol ${ }^{4}$ to calculate dose distributions around the HDR source, and to plan the necessary treatment. Since the treatment relies on the correct delivery of the plan by the HDR remote afterloader, it is 
desirable to develop an independent quality assurance (QA) method that accurately verifies the HDR source dwell positioning and timing pattern for each catheter. A sound QA program should include a pre-treatment plan verification ${ }^{5}$ and would give confidence of a correct execution at the time of treatment. Although the afterloader has high precision in its control of the source movement, treatment outcomes of plans with multiple catheters are more susceptible to deviations in dwell position and time ${ }^{6}$.

There have been efforts found within the literature to develop a method for verification of planned dwelling and timing patterns before and during treatment. These include pinhole imaging ${ }^{7,8}$, diamond detectors $^{9}$, flat panel $^{10}$ and EPIDs ${ }^{11}$, film and photodiodes ${ }^{12}$, ion chamber arrays ${ }^{13}$ and video cameras ${ }^{*}$. These devices, while capable of source tracking, can be costly and are limited in their ability to perform a quick, comprehensive and automated analysis of a full treatment plan.

The feasibility study of a two dimensional detector array "magic plate", housed inside a "magic phantom" (MPh), showed the "magic plate" to be capable of determining the source position within three dimensions with a fast acquisition speed $(0.1-100 \mathrm{~ms})^{14}$. This work seeks to evaluate the proof of concept performance of the novel $\mathrm{MPh}$, with updated electronics and software toolkit, as a complete system for HDR pre-treatment plan and afterloader verification. We aim to show that the $\mathrm{MPh}$ has the potential to accurately measure the dwell positions and times of any treatment, compare those measured values against the treatment plan, determine any errors in delivery, and estimate the total dose delivered - including the transit dose contribution along the "magic plate" plane.

The MPh system was CT scanned and using ONCENTRA ${ }^{\circledR}$ TPS (Nucletron, the Netherlands), two 20 catheter treatment plans were created. The treatment plans were measured using the MPh and the dwell positioning and timing were evaluated based on a new acceptance criterion for HDR QA, named the position-time gamma index. Dose distributions were calculated by the developed software,

\footnotetext{
" Mick Radio-Nuclear Instruments, Inc., Mount Vernon, NY, USA, accessed: $11^{\text {th }}$ of August 2014 (available URL: http://www.micknuclear.com/home/products/quality_assurance_tools.html)
} 
and compared to the treatment plan and EBT3 film measurements using the $2 \mathrm{D}$ gamma analysis proposed by Low et $a l^{15}$.

\section{Materials}

The "magic phantom", MPh, is the HDR specific casing for the silicon-based detector system "magic plate". The "magic plate" consists of 121 p-type silicon epitaxial diodes in an $11 \times 11$ array, and has been characterized for use in IMRT/VMAT radiation therapy ${ }^{16}$ and HDR brachytherapy ${ }^{14}$. Both the "magic plate" and "magic phantom" were developed at the Centre for Medicial Radiation Physics (CMRP), University of Wollongong, Australia.

The silicon epitaxial diodes were characterized previously and demonstrated a high degree of radiation hardness, up to $120 \mathrm{kGy}$ of water equivalent absorbed dose from a ${ }^{60} \mathrm{Co}$ source, and a stable response within $5 \%$ after $40 \mathrm{kGy}^{17}$. The "magic plate" used for experiments was pre-irradiated to 40 $\mathrm{kGy}$, and the response was normalized prior to testing using a $20 \times 20 \mathrm{~cm}^{2}$ photon field from a $6 \mathrm{MV}$ medical linac at a source-to-surface distance of $100 \mathrm{~cm}$ and placed at depth of $10 \mathrm{~cm}$ inside of a $30 \times 30 \times 30 \mathrm{~cm}^{3}$ Solid Water phantom. The dose profile was verified to be flat as part of the linac QA using ion chambers.
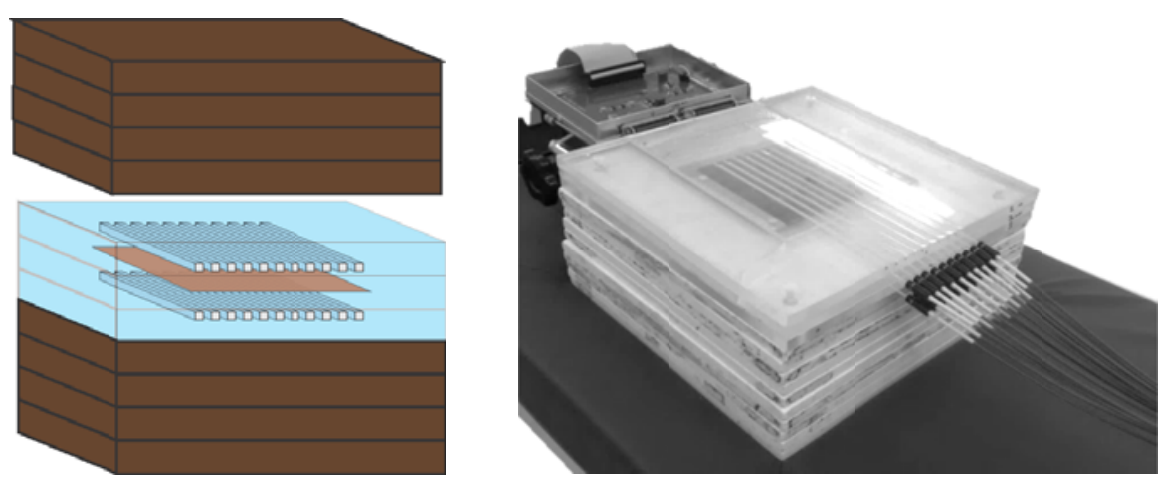

Figure 1 - Illustration and photo of the "magic plate" inside the MPh, with slabs of Solid Water above and below. On the right, the HDR catheters within the MPh are seen to be attached to the HDR afterloader transfer tubes.

The MPh consists of three $1 \mathrm{~cm}$ slabs of PMMA plastic, with an area of $30 \times 30 \mathrm{~cm}^{2}$. PMMA was chosen as material for this generation of the MPh due its optical transparency, which assisted with preliminary positional calibrations. It is noted that while this material is not well suited for 
dosimetry $^{18,19,20}$, the source tracking method is based on the detectors response within this phantom and has been previously characterized ${ }^{14}$. Later versions will be completely made of Solid Water. An illustration and photo are seen in Figure 1.

The "magic plate" was inserted into the middle slab of the $\mathrm{MPh}$, placing the $10 \times 10 \mathrm{~cm}^{2}$ field of view (FOV) of the detectors directly in the center of the phantom. The top and bottom layers have ten $15 \times 2 \times 2 \mathrm{~mm}^{3}$ channels machined onto the boundary to the center slab, allowing for the insertion of 20 HDR plastic catheters. A thickness of $13.5 \mathrm{~cm}$ of water equivalent material was placed both above and below the $\mathrm{MPh}$, to ensure backscattering conditions were met.

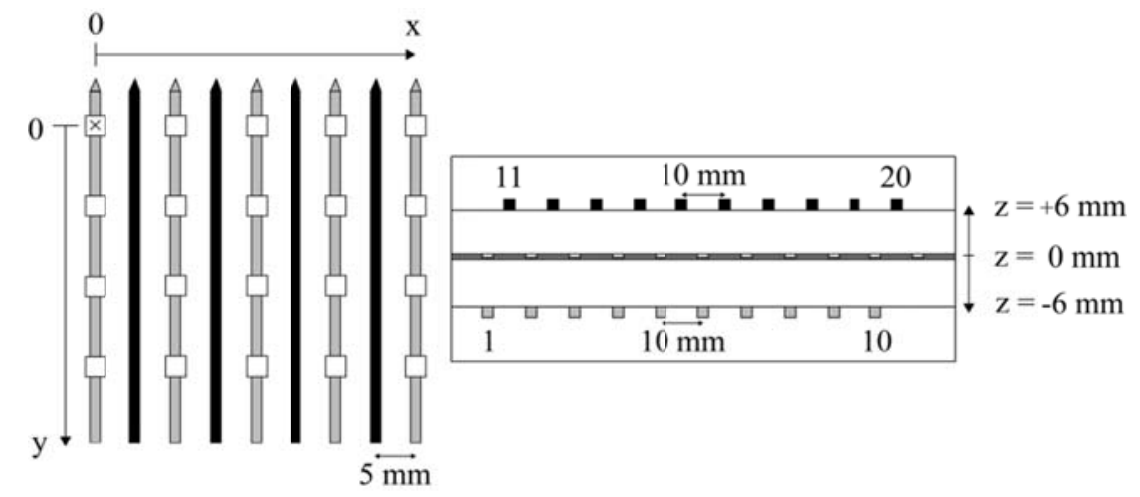

Figure 2 - Illustration of catheter channels with respect to the "magic plate". White squares represent diode positions relative to catheters with the two rows of catheters above (black), and below (grey). The left shows a top view of the catheters inserted into the MPh with respect to the detectors position and the $\mathrm{x}$ and $\mathrm{y}$-coordinate origin on a corner detector, as indicated by a cross. The right shows the side view of the MPh with the catheters numbered 1 to 20 .

Two rows of 10 catheters were inserted into the trenches approximately $6 \mathrm{~mm}$ above and below the "magic plate" array. The catheter trenches were spaced at $10 \mathrm{~mm}$ apart with the above row placed between the columns of the MPh detector array, and the below row directly aligned with the detector columns, shown in Figure 2. Using a corner detector as the origin of the MPh coordinate system, indicated by a cross inside the detector, the catheters are at $\mathrm{z}= \pm 6 \mathrm{~mm}$, and at an $\mathrm{x}$-coordinate corresponding to the catheter columns, with the catheter length along the y-coordinate. Using the result of the source tracking algorithm at each frame of measurement, the $x-y-z$ coordinate measurement of the source was translated to catheter number and position within the catheter. 
All $121 \mathrm{MPh}$ detectors were read out by an in-house designed front-end electronics system, named “AFE data acquisition system" (AFE DAQ) ${ }^{21,22}$. The system uses two Texas Instrument commercial electrometer AFE-0064 chips and is controlled by a CMRP designed FPGA master board. The timing of the system is accurately governed by a $20 \mathrm{MHz}$ master clock on the FPGA board and has an estimated error in timing of $50 \mathrm{~ns}$. This communicates to the PC via USB2.0, and is controlled by an in-house designed firmware. The AFE DAQ is capable of a variable integration time (between 14 to $9900 \mu$ s), with a stable sampling frequency of 0.1 to $10 \mathrm{kHz}$, and allows for triggering from external sources for linear accelerator measurements or using an internal trigger signal. For this work, a $1 \mathrm{kHz}$ sampling frequency and $100 \mu$ s integration time was used for all measurements.

A custom software interface was developed in $\mathrm{C}++$ to: a) communicate with the FPGA and drive the AFE DAQ; b) perform source tracking in real time and in post-processing; c) perform dwell position and timing analysis; and d) calculate the absorbed dose from the TG-43U1 protocol based on measured source dwelling position and time and compare it to the dose prescribed by the treatment plan. The system and software measures the charge generated in all $121 \mathrm{MPh}$ detectors within the integration time, at a periodic sampling frequency. The response at each measurement frame for each detector was collected in a data file for post-processing.

The software performs TG-43U $1^{4}$ dose calculations to predict the dose generated to the MPh plane, based upon the measured dwell position analysis, the HDR ${ }^{192}$ Ir Flexisource specifications ${ }^{23}$ and known source activity. This was compared to the dose predicted by the TPS for selected points, and dose maps were generated and verified experimentally using Gafchromic EBT3 film. Due to the manufacturers stated dose range of " $1 \mathrm{cGy}$ to $>40 \mathrm{~Gy}$ ", the tested dose range of up to $90 \mathrm{~Gy}^{24}$ and relatively weak energy dependence for energies as low as $50 \mathrm{keV}^{25,26}$, the use of Gafchromic EBT3 appears well suited for use in ${ }^{192} \operatorname{Ir}$ HDR dosimetry and plan verification.

\footnotetext{
${ }^{\dagger}$ Ashland International Specialty Products (ISP) Advanced Materials, New Jersey, USA, Gafchromic EBT3 product brochure (2011).
} 
The MPh was imaged using the $\mathrm{CT}$ scanner, and the images imported into the Nucletron ONCENTRA ${ }^{\circledR}$ TPS. Each small MPh detector element could be seen on the 3D image reconstruction, with each detector selected as a point for dose calculations. The source was programmed to dwell at various positions and times inside each of the 20 catheters to create a nonspecific treatment plan with a maximum dose of $600 \mathrm{cGy}$ to a single detector element. Each catheter had varying dwell positions and times arranged in the plan in an unsystematic fashion.

A copy of the plan was created and modified by a member of the hospital medical physics staff, without the supervision of the authors, and an undisclosed number of changes were made. The plan was altered by the inclusion of new dwell points, points shifted or removed, and dwell times lengthened or shortened, to simulate a scenario where there is a misadministration by the afterloader. Each plan was exported as a DICOM file, preserving the source dwell and timing information for each catheter and the calculated absorbed dose at each detector position on the MP. Both DICOM plan files were subsequently read by the custom software suite; first to compare the measured dwelling position-time pattern with the original plan and identify the modifications using the position-time gamma analysis, and then to verify that all alterations measured were indeed made within the modified plan. All plans were delivered using the Flexitron HDR afterloader (Nucletron, the Netherlands) at the St George Cancer Care Centre, Sydney.

\section{Methods}

\section{A. Determination of source dwell positions and dwell times}

A method for determining the HDR ${ }^{192}$ Ir source position using a two dimensional detector array was introduced within the "magic plate" feasibility study ${ }^{14}$. It is based on the sampled dose rate measurements from the nine neighboring diodes with the largest signals, followed by an iterative optimization procedure that takes into account the angular response of the diodes ${ }^{27}$. This method calculates, for each frame of measurement, the $x-y-z$ coordinates of the HDR source when in the FOV of the MPh detectors. Building upon these results, the software interface was designed to automatically determine the source dwell positions and dwell times from the treatment measurement. 
Time gaps between the last position of each catheter and the first dwell of the next catheter do not affect the source position determination, as there is extremely little signal when the source is outside of the phantom. When the maximum signal during a measurement frame is below a predetermined threshold level, the software does not execute the source position calculation algorithm. The threshold level is dependent on the expected range of source activity and corresponds to the estimated current generated within a detector by a source at a distance of $150 \mathrm{~mm}$ away in the $\mathrm{z}$ direction, inside the FOV of the MPh. The noise level is very low in this developed system, and consequently, has minimal bearing on measurements.

The unmodified 20 catheter plan delivery was measured using the MPh and the source position was analyzed in post-processing. For each catheter, a position-frequency histogram was generated from the source tracking data. The sampled source position was binned and peaks appeared in the histogram when many measurements occurred at the same position, i.e. when the source was dwelling. The number of counts under each peak of the histogram corresponds to the measured dwell time, as the time for each count was dependent on the sampling frequency of the system. The dwell position and uncertainty was determined by the center and full width half maximum (FWHM) of the peak. These results were compared to the dwell position and times found within the treatment planning file.

The position of the source is related to the tip of each catheter, which is registered in the frame of the $\mathrm{MPh}$ with coordinate system origin based on a left corner detector. A position calibration test, relative to the $\mathrm{MPh}$ origin, was performed by driving the source at multiple positions within a single catheter and simultaneously measuring with the MPh and exposing EBT3. The EBT3 film was registered to the MPh by fixing it rigidly between the "magic plate" and top catheter plane. The center of mass of each source image on the film and the dwell positions calculated by the software were compared with the planned dwell position. The planned dwell positions were based on the CT scan of the MPh with tip markers inside each catheter.

\section{B. Determination of the minimum measurable step size and dwell time}


The Flexitron HDR afterloader can be programmed to drive the HDR source using varying step sizes and dwell times, with a minimum of $1 \mathrm{~mm}$ and $0.1 \mathrm{~s}$, respectively. To be feasible for plan verification, the MPh system must be able to determine dwell positions and times accurately, over this range of step sizes and dwell times.

To assess the dwell position resolution of the system, the afterloader was programmed to deliver the HDR source through a single catheter, moving with progressively reduced step sizes down to $1 \mathrm{~mm}$. Each dwell position had a programmed dwell time of $3 \mathrm{~s}$ and was measured using the MPh system. The transit time of the source from one dwell position to the next was also measured.

To assess the dwell time resolution of the system, the HDR afterloader was programmed to drive the source to 3 dwell positions within a single catheter for the same amount of time. The measurement was repeated for a range of dwell times from $10 \mathrm{~s}$ down to $0.1 \mathrm{~s}$.

\section{C. Position-time gamma index}

To quantitatively compare the source position and dwell times within the catheter to the treatment plan, a new metric is introduced based upon the dose-position gamma index. The use of the 2D gamma index in QA was first described by Low et $a l^{15}$ and was used to quantitatively compare dose distributions for external beam radiation therapy. It compares the dose difference, point-by-point, and the distance-to-agreement between the evaluated dose distribution and that of the reference dose distribution, and is used for analysis in sections 3.E and 4.E.

Adopting the formalization of this method and applying it to HDR brachytherapy, it is possible to compare the measured treatment to the plan by evaluating the source dwell and timing patterns. By using acceptable distance-to-agreement and time-to-agreement criteria values, a pass-fail grade (pass if gamma value is equal to or less than 1) is determined for all measured dwell positions and times.

Table 1 - Definition of symbols used for HDR position-time gamma index.

\begin{tabular}{|c|c|c|}
\hline Symbol & Equation & Description \\
\hline$A^{n}\left(x_{i A}, t_{i A}\right)$ & N/A & The $i$-th dwell position, $x_{i A}$, and dwell \\
\hline
\end{tabular}




\begin{tabular}{|c|c|c|}
\hline & & time, $t_{i A}$, of set $\mathrm{A}$ within catheter $\mathrm{n}$. \\
\hline$B^{n}\left(x_{j B}, t_{j B}\right)$ & $\mathrm{N} / \mathrm{A}$ & $\begin{array}{l}\text { The } j \text {-th dwell position, } x_{j B} \text {, and dwell } \\
\text { time, } t_{j B} \text {, of set B within catheter } \mathrm{n} \text {. }\end{array}$ \\
\hline$\Delta x$ & N/A & Distance-to-agreement criterion (DTA). \\
\hline$\Delta t$ & $\mathrm{~N} / \mathrm{A}$ & Time-to-agreement criterion (TTA). \\
\hline$\delta_{i j}^{n}\left(x_{i A}, x_{j B}\right)$ & $\delta_{i j}^{n}\left(x_{i A}, x_{j B}\right)=\left|x_{i A}-x_{j B}\right|$ & $\begin{array}{l}\delta_{i j}^{n} \text { is the spatial difference between the } \\
i \text {-th dwell position of set A and the } j \text {-th } \\
\text { dwell position of set B for catheter } n \text {. }\end{array}$ \\
\hline$\tau_{i j}^{n}\left(t_{i A}, t_{j B}\right)$ & $\tau_{i j}^{n}\left(t_{i A}, t_{j B}\right)=\left|t_{i A}-t_{j B}\right|$ & $\begin{array}{l}\tau_{i j}^{n} \text { is the difference between the } i \text {-th } \\
\text { dwell time of set A and the } j \text {-th dwell } \\
\text { time of set B for catheter } \mathrm{n}\end{array}$ \\
\hline$\Gamma_{i j}^{n}\left(\delta_{i j}^{n}, \tau_{i j}^{n}\right)$ & $\Gamma_{i j}^{n}\left(\delta_{i j}^{n}, \tau_{i j}^{n}\right)=\sqrt{\left(\frac{\delta_{i j}^{n}\left(x_{i A}, x_{j B}\right)}{\Delta x}\right)^{2}+\left(\frac{\tau_{i j}^{n}\left(t_{i A}, t_{j B}\right)}{\Delta t}\right)^{2}}$ & $\begin{array}{l}\text { Generalized } \Gamma \text { index computed for all } \\
\text { dwell positions and times of set } A \text { and } \\
\text { set } B \text { for catheter } \mathrm{n} \text {. }\end{array}$ \\
\hline$\gamma^{n}\left(x_{i A}\right)$ & $\gamma^{n}\left(x_{i A}\right)=\min \left\{\Gamma_{i j}^{n}\left(\delta_{i j}^{n}, \tau_{i j}^{n}\right)\right\} \forall\left\{B^{n}\left(x_{j B}, t_{j B}\right)\right\}$ & $\begin{array}{l}\text { The } \gamma \text { index - the minimum generalized } \\
\qquad \Gamma \text { for the set } \mathrm{B}, B^{n}\left(x_{j B}, t_{j B}\right) \text { dwell } \\
\text { positions and dwell times within catheter } \\
\qquad \mathrm{n} \text {. }\end{array}$ \\
\hline
\end{tabular}

The formalization of this method is found in Table 1. Two sets, A and B, represent the measured and TPS dwell position timing patterns within catheter $\mathrm{n}$. The gamma index compares the dwell positions within set $\mathrm{B}$ against those in set $\mathrm{A}$. The gamma index was calculated twice; first to compare the treatment measured by the MPh (set B) against the treatment plan (set A), and then to compare the treatment plan (set B) against the measured (set A). This increases the robustness of the method, taking into account cases when sets $\mathrm{A}$ and $\mathrm{B}$ have unequal numbers of dwell positions due to positions introduced or missed by the afterloader. A graphical representation of the position-time criteria is seen in Figure 3. 


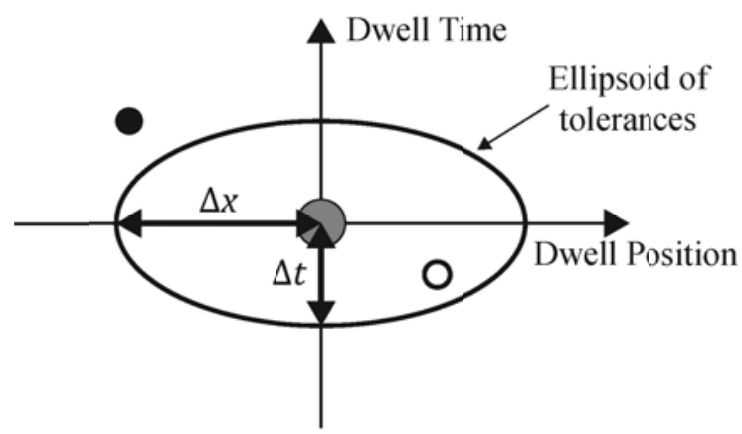

Reference Dwell Point

Dwell Point within tolerance

Dwell Point outside tolerance

Figure 3 - Geometric representation of the position-time criteria. Dwell points inside or on the ellipssoid have a gamma value equal or less than 1 and pass the criteria. Outside this ellipsoid, the gamma index value is greater than 1 and fails the criteria.

\section{D. Position-time gamma index on modified plan}

The modified version of the plan was measured by the MPh and the results were compared to the original plan. The modified plan had an undisclosed number of changes made by medical physicists to simulate an incorrect treatment delivery. This was tested to verify the position-time gamma index analysis method. Following the software analysis of the measurement, the modified treatment plan DICOM file was read to verify that all the alterations were correctly identified.

\section{E. Comparisons between "magic phantom" calculated dose, TPS and EBT3 film dose measurements}

The software suite performs dose calculations based upon the TG-43U1 formalism, and was tested to ensure that calculations agreed with doses prescribed by the TPS. Each MPh detector coordinate was selected for point dose calculations and stored inside the DICOM file, along with the source dwell positions and times. A direct comparison between the values within the DICOM was performed for 121 points using the dose calculation engine of the software.

Gafchromic EBT3 film (Ashland ISP Advanced Materials, USA) lot \#A07151302 was used to verify the dose calculated by the TPS and by the custom software suite. Prior to this measurement, the film was calibrated by exposing five $10 \times 10 \mathrm{~cm}^{2}$ pieces to the HDR ${ }^{192} \mathrm{Ir}$ source. The MPh was 
disassembled and the top layer with catheter trenches was placed within a $30 \times 30 \times 30 \mathrm{~cm}^{2}$ Solid Water stack. A single piece of film was placed at the center of the stack, and a single catheter was inserted into a trench directly over the film, at an approximate source-to-film distance of $16 \mathrm{~mm}$. ONCENTRA ${ }^{\circledR}$ TPS was used to calculate the necessary time to deliver a dose to the center of the film, with the 5 pieces of film irradiated to $100,200,400,600$ and 1000 cGy using a single dwell position.

All pieces of film were pre-scanned and post-irradiation scanned using a 48-bit RGB transmission film scanner, the EPSON 10000XL, at a resolution of 300 dpi, with no color or sharpness corrections and at a consistent orientation and position to the scanner. Approximately 72 hours passed between film irradiation and scanning to ensure the film response had stabilized. The calibration films were scanned and the image files were measured using IMAGEJ (version 1.47v). Six consecutive scans were performed for each piece of film to ensure consistent performance of the scanner on the last three used for analysis. A calibration curve was generated for the red color channel based upon the largest change in optical density and exposed dose. The total uncertainty was calculated to be approximately $6.1 \%$ (1 S.D), based upon an uncertainty budget which considers the source-to-film position, scanner inhomogeneity and reproducibility and the TPS dose calculations and source activity measurements.

Using the software, dose maps were generated along the MPh detector plane for the original treatment plan, the measured treatment based upon the measured dwell positions and times, and for the modified treatment plan. The original treatment plan was then measured by replacing the "magic plate" detector with EBT3 film. The film was cut to match the size of the "magic plate" and markings were made for alignment. Both the film and "magic plate" were later scanned using the scanner, so that the film dose map positions could be registered to those calculated by the MPh software. The change in optical density was converted to dose using the calibration curve. The dose maps were then compared using the 2D gamma analysis, with varying dose difference and distance-to-agreements criteria. Each map had a size of $10 \times 10 \mathrm{~cm}^{2}$, with pixel dimensions of $0.5 \times 0.5 \mathrm{~mm}^{2}$. 
The total transit dose for the MPh diode positions was also estimated by performing dose calculations for each measured source position between dwell positions, assuming a discrete source movement between consecutive frames.

\section{Results}

\section{A. Determination of source dwell positions and dwell times}

Figure 4 shows the calculated source position and dwell time in a position within the first catheter verses time, determined by the algorithm described in detail in previous study ${ }^{14}$. It can be seen that the source travels from the afterloader and dwells at the furthest dwell position away from the catheter tip, and moves progressively closer. The TPS plan for this catheter is graphed for comparison and shows good agreement for positions larger than $5 \mathrm{~mm}$ from the coordinate origin based on the MPh corner detector. While the TPS does not take into account the motion of the source in transit between dwell points, it is possible to measure this due to the high speed of the MPh readout system.

The last two dwell positions were close to the catheter tip and the edge of the FOV of the "magic plate", and used fewer detectors for calculation of source position. As the source tracking algorithm is based upon the agreement of the detector with the largest response and its neighbors, reduced numbers of detectors can result in a shift in true position. For accurate source dwell position calculations, the TPS plan should be configured to set the last dwell points at least 5-10 $\mathrm{mm}$ away from the edge of the MPh FOV. While there is a reduction in useable catheter length for this particular design of "magic plate", it will be possible to measure the dwell position accurately within a range of 80-90 $\mathrm{mm}$. This issue was considered for the design of the next generation of the "magic plate", MP512, which features a larger area, 512 diodes and a $5 \mathrm{~mm}$ pitch between detectors. 


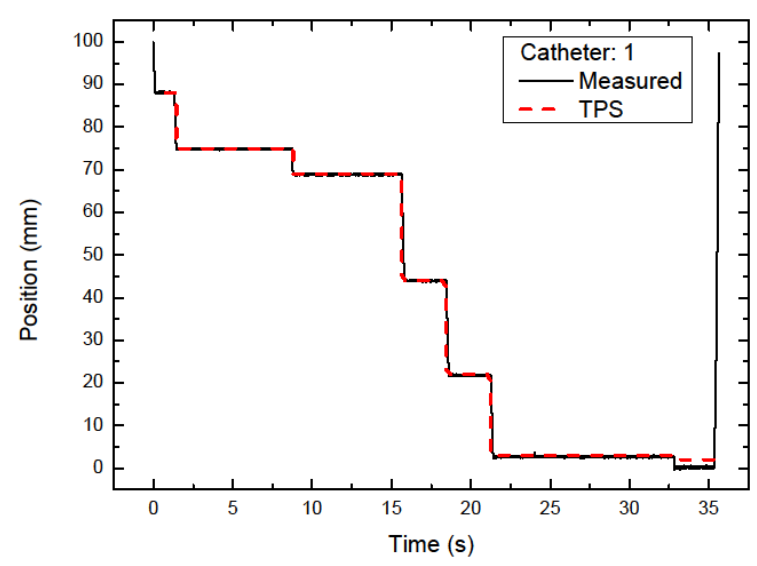

Figure 4 - Source tracking measurement and TPS plan for Catheter 1. The position calculated was relative to the MPh coordinate system.

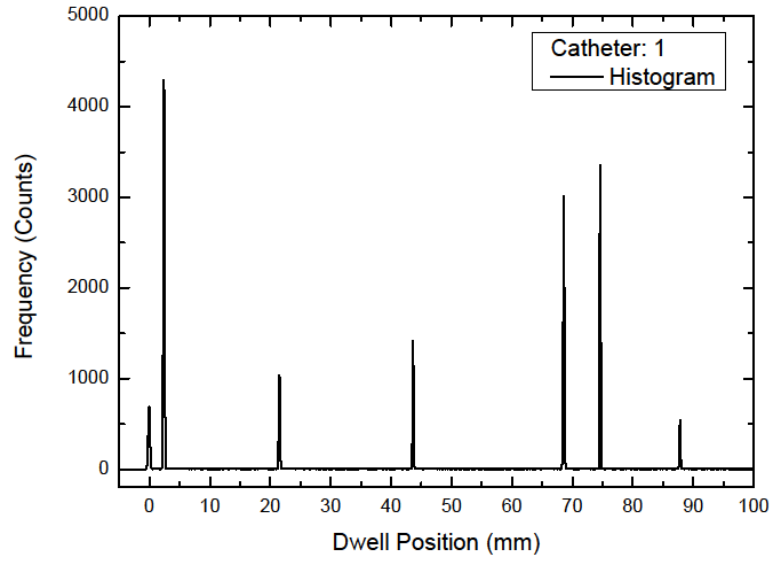

Figure 5 - Measured dwell position frequency histogram for Catheter 1. The net area of each peak corresponds to the total dwell time at the position. Each count represents $1 \mathrm{~ms}$ due to measurement frequency.

A position frequency histogram was generated from the source tracking calculations for each catheter. Each histogram had a bin width of $0.1 \mathrm{~mm}$ and the counts of source position were related to the electronic sampling frequency. Each count within the histogram represented $1 \mathrm{~ms}$ of time that the source had spent at that calculated position. Figure 5 shows the histogram for Catheter 1. Each dwell point was seen to be a narrow peak, where the center was equal to the dwell position and the FWHM was the uncertainty of position. The number of counts converted to time under each peak is equal to the total dwell time for that dwell position.

The calculated and TPS planned dwell position-time patterns for catheter 1 are also presented in Figure 6. Uncertainties in the dwell positions were typically of the order of $0.2 \mathrm{~mm}$ (2 S.D) and are unable to be seen on this graph. Good agreement between these dwell patterns were seen for the majority of points, except for those close to $0 \mathrm{~mm}$, due to the edge of FOV effect. The dwell positions and times were compared to the treatment plan. 


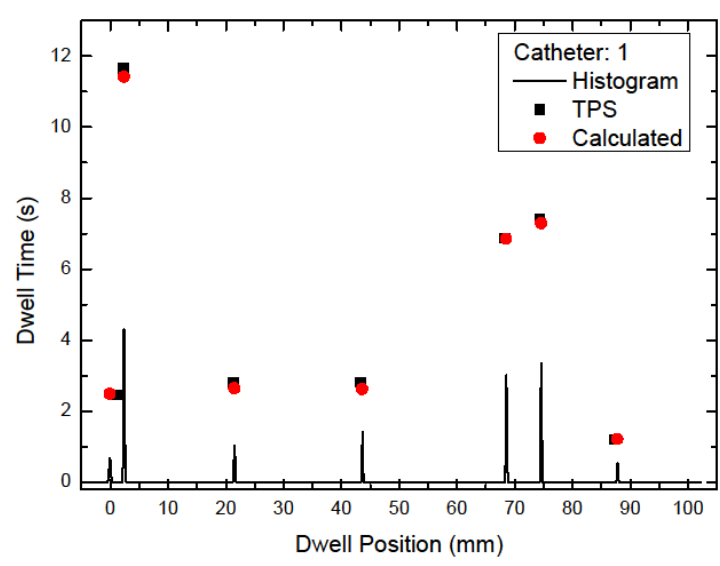

Figure 6-Dwell position frequency histogram and comparison of dwell position timing pattern for Catheter 1 with the TPS plan. The circles show the total dwell time calculated from the corresponding peak at that position.

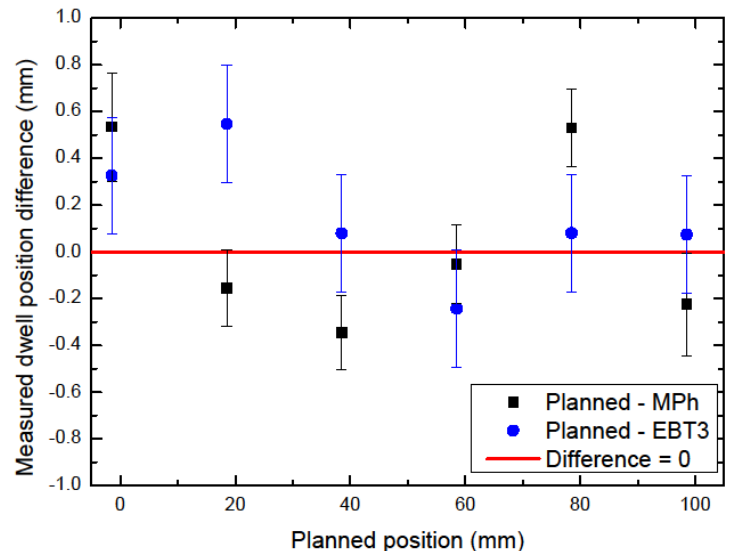

Figure 7 - Difference between the measured and planned dwell positions for the MPh calculation and EBT3 film for single catheter measurement.

Figure 7 shows the difference along the catheter length between the measured and planned dwell positions for the position calibration test. The source was driven through a single catheter towards the catheter tip and the EBT3 film was irradiated, while the MPh measured. Six dwell positions were identified by both the software and the film analysis with all differences less than $0.8 \mathrm{~mm}$. An uncertainty of $\pm 0.25 \mathrm{~mm}$ (2 S.D) was estimated due to the coordinate registration of the film to the MPh reference frame.

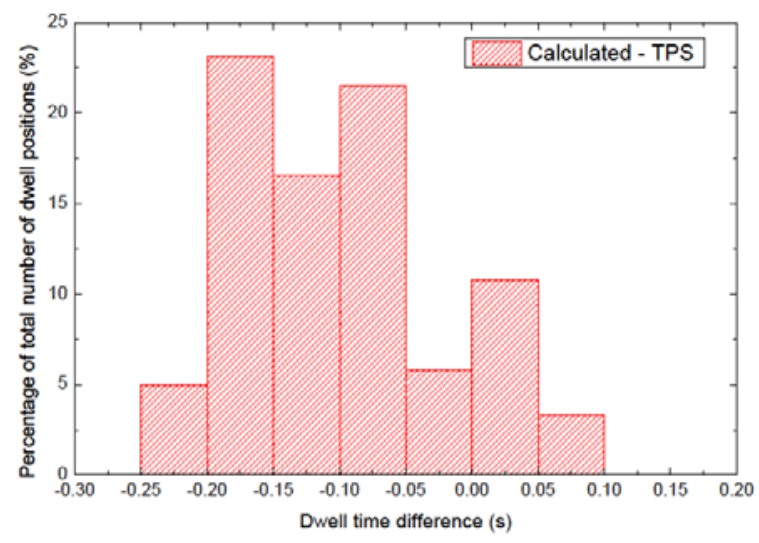

Figure 8 - Histogram showing the difference in dwell times of the Measured and TPS.

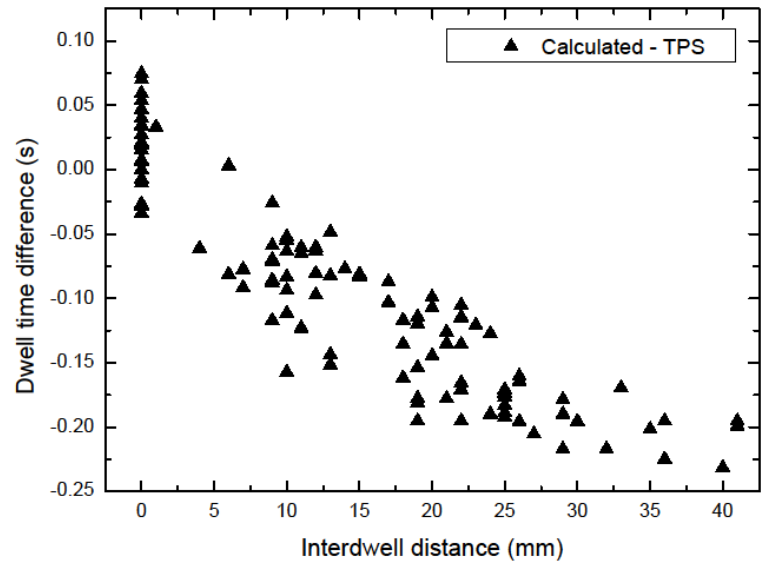

Figure 9 - Difference between measured and planned dwell times against interdwell distance.

The average difference between the measured dwell position and the TPS plan for $86 \mathrm{dwell}$ positions was $0 \pm 0.63 \mathrm{~mm}$ (2 S.D), taking into account the larger differences due to the edge of FOV effect. 
Seen in Figure 8, the dwell time differences were found to be less than $0.25 \mathrm{~s}$, and the majority showed that the MPh measured less than the expected planned dwell times. It is expected that due to the highly accurate internal clock of the electronics used, the error in timing is extremely small.

This difference in dwell time is most likely due to a feature of the Flexitron afterloader that reduces dwell times to compensate for additional dose delivered while the source is in transit between successive dwell points. In Figure 9, the differences in dwell times are plotted against the interdwell distances (the distance between two dwell positions), showing that the difference in dwell time for the subsequent position is proportional to the distance the source had to travel. An interdwell distance of 0 $\mathrm{mm}$ represents the first dwell position within the catheter, where it is expected that there will be no change in dwell time.

According to the ONCENTRA ${ }^{\circledR}$ MasterPlan Physics and Algorithms manual ${ }^{\ddagger}$, it is assumed that this afterloader drives the source at a speed of $50 \mathrm{~cm} / \mathrm{s}$ and for an interdwell distance of $50 \mathrm{~mm}$ the dwell time is reduced by a maximum of $0.1 \mathrm{~s}$. It is noted that this feature may not be seen in some afterloaders. The measured dwell time differences are larger than expected but, as seen in the feasibility study for the "magic plate" detector, the average source speed was measured and found to vary from 12.5 to $37.5 \mathrm{~cm} / \mathrm{s}$ based on interdwell distance ${ }^{14}$. Although a different afterloader was used for the feasibility study, if it is assumed they share similar average source speeds, the variation in dwell time difference can be justified.

\section{B. Determination of the minimum measurable interdwell distance and dwell time}

The HDR source was driven to 9 dwell positions in a single catheter, with spaces between dwell positions varying from $30 \mathrm{~mm}$ to $1 \mathrm{~mm}$. As seen in Table 2, the dwell positions were determined by the MPh, and the system was capable of resolving the dwell positions separated by $1 \mathrm{~mm}$. There is some deviation noted between dwell positions, which may be due to the edge of FOV effect

\footnotetext{
* Oncentra MasterPlan v3.2, Physics and Algorithms Manual, 192.739ENG-02, Nucletron, the Netherlands.
} 
previously discussed. The dwell times for each position were found to follow the trend of reduced times, as a function of interdwell distance (Figure 9) and agreed with the determined transit times.

Table 2 - Comparison of the planned position in an HDR catheter and the position and time measured using the MPh.

\begin{tabular}{|c|c|c|c|}
\hline Planned position $(\mathrm{mm})$ & Measured position $(\mathrm{mm})$ & $\begin{array}{c}\text { Measured dwell time (s) } \\
( \pm 0.001 \mathrm{~s})\end{array}$ & $\begin{array}{c}\text { Measured transit time (s) } \\
( \pm 0.001 \mathrm{~s})\end{array}$ \\
\hline 83.6 & $83.49 \pm 0.14$ & 3.015 & 0.219 \\
\hline 53.6 & $53.82 \pm 0.12$ & 2.792 & 0.172 \\
\hline 33.6 & $34.23 \pm 0.14$ & 2.849 & 0.109 \\
\hline 23.6 & $23.95 \pm 0.18$ & 2.919 & 0.066 \\
\hline 18.6 & $18.27 \pm 0.16$ & 2.946 & 0.040 \\
\hline 14.6 & $15.06 \pm 0.16$ & 2.987 & 0.054 \\
\hline 11.6 & $11.54 \pm 0.16$ & 2.976 & 0.041 \\
\hline 9.6 & $8.72 \pm 0.18$ & 2.989 & 0.027 \\
\hline 8.6 & $7.35 \pm 0.16$ & 2.991 & \\
\hline
\end{tabular}

The minimum measurable dwell time for the MPh system was evaluated by using three dwell positions, at $13.6 \mathrm{~mm}, 18.6$ and $28.6 \mathrm{~mm}$ from the catheter tip, and varying the planned times. Each position was set to have the same dwell time, but these varied when measured, except for Position 1 which was the first the source was driven to. In Table 3, it can be observed that the minimum dwell time that was measured was $0.067 \pm 0.001 \mathrm{~s}(2 . \mathrm{SD})$. Following the trend of dwell time reduction, for the planned dwell times of $0.1 \mathrm{~s}$, Position 2 was expected to have a time of 0.01-0.02 s, but could not be detected by the system due to not being distinguishable within the position frequency histogram.

Table 3 - Determination of the minimum measurable dwell time

\begin{tabular}{|c|c|c|c|}
\hline Planned dwell time (s) & \multicolumn{3}{|c|}{ Measured dwell time (s) ( $\pm 0.001 \mathrm{~s})$} \\
\hline TPS & Position 1 (28.6 mm) & Position 2 (18.6 mm) & Position 3 (13.6 mm) \\
\hline 10 & 10.012 & 9.914 & 9.955 \\
\hline 5 & 5.015 & 4.908 & 4.951 \\
\hline 3 & 3.014 & 2.898 & 2.955 \\
\hline
\end{tabular}




\begin{tabular}{|c|c|c|c|}
\hline 2 & 2.014 & 1.911 & 1.951 \\
\hline 1 & 1.013 & 0.916 & 0.957 \\
\hline 0.5 & 0.512 & 0.414 & 0.458 \\
\hline 0.3 & 0.315 & 0.213 & 0.155 \\
\hline 0.2 & 0.212 & 0.109 & 0.067 \\
\hline 0.1 & 0.107 & Unable to determine & \\
\hline
\end{tabular}

The verification of the accuracy of all dwell times set by the TPS is impossible, as the afterloader always modifies the actual dwelling time by taking the transit time into account. We have, however, demonstrated an obvious relationship between the absolute dwell time and interdwell distance, which is noticeable for all dwell times. This shows that the timing capabilities of this developed system surpass the programmable limitations of the afterloader system.

\section{C. Position-time gamma analysis}

The software suite determines the dwell position and times for all the catheters and compares them against the treatment plan using the position-time gamma analysis. By applying the equations described in Table 1 to the dwell pattern of Catheter 1, the gamma index values for each dwell position were calculated and are shown in Figure 10. Taking into account the differences between the measured dwell position and times from section 4.A, the DTA and TTA were set to $1.3 \mathrm{~mm}$ and $0.3 \mathrm{~s}$, respectively. Uncertainties were calculated based on the standard deviation of the dwell position measurement and the DTA criteria. 


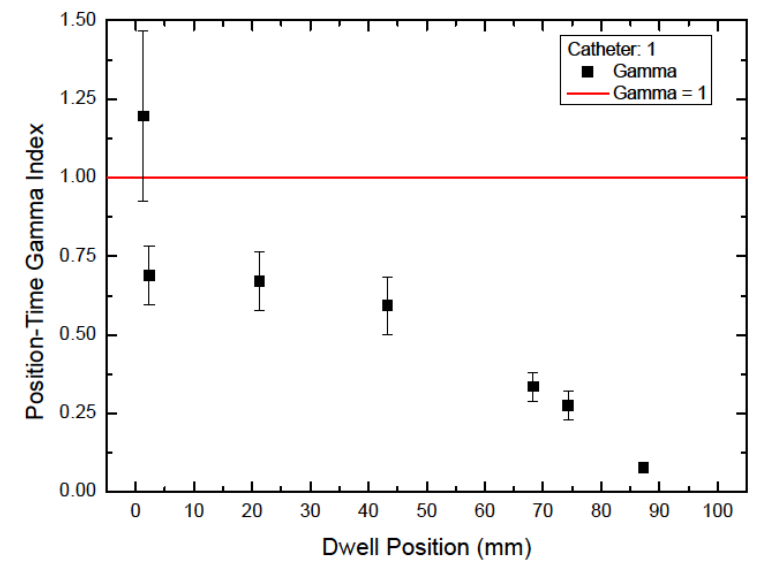

Figure 10 - Gamma analysis for Catheter $1(\mathrm{DTA}=1.3 \mathrm{~mm}$, $\mathrm{TTA}=0.3 \mathrm{~s})$.

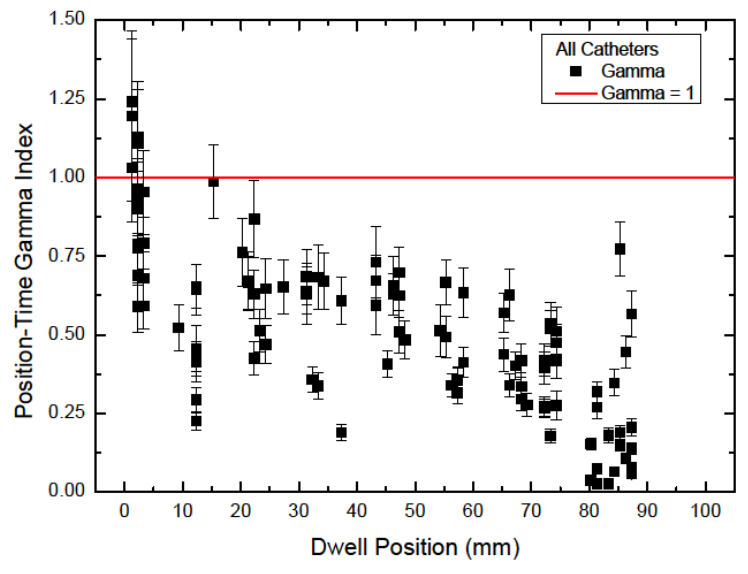

Figure 11 - Gamma analysis for all catheters $($ DTA $=1.3$ $\mathrm{mm}, \mathrm{TTA}=0.3 \mathrm{~s})$.

Applying the gamma index to all 20 catheters, it can be seen from Figure 11 that the majority of the treatment $(95 \%)$ have gamma values of less than or equal to 1 . There are 5 points with gamma values greater than 1, but they are considered to pass, due to their uncertainty.

\section{D. Position-time gamma index on modified plan}

The modified plan was measured using the MPh and using the results from the position-time gamma analysis, an estimated 11 changes were expected to have been made to the treatment plan. One change to Catheter 3, seen in Figure 12, was the addition of a dwell position. This position was seen to be at $17.2 \pm 0.1 \mathrm{~mm}(2 \mathrm{~S} . \mathrm{D})$ from the coordinate origin, with a dwell time of $14 \mathrm{~s}$. 


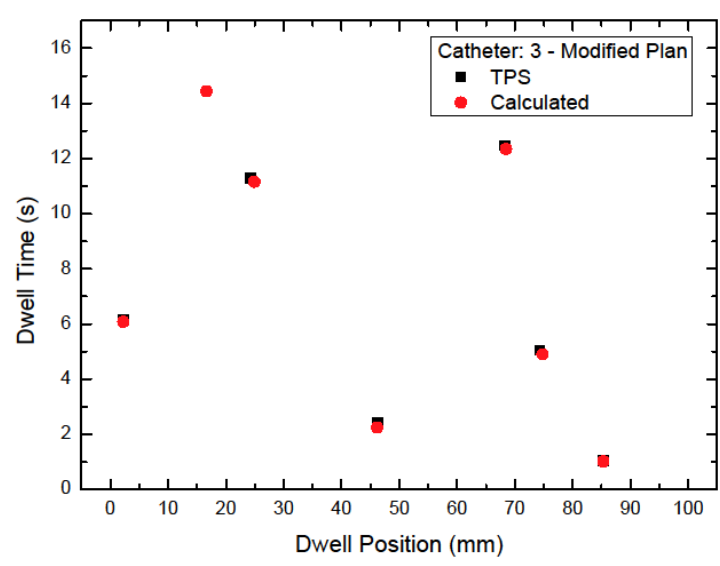

Figure 12 - Comparison of dwell pattern for Catheter 3 against the TPS plan. It can be seen that the MPh measures a dwell position at $17.0 \mathrm{~mm}$ introduced within the modified plan.

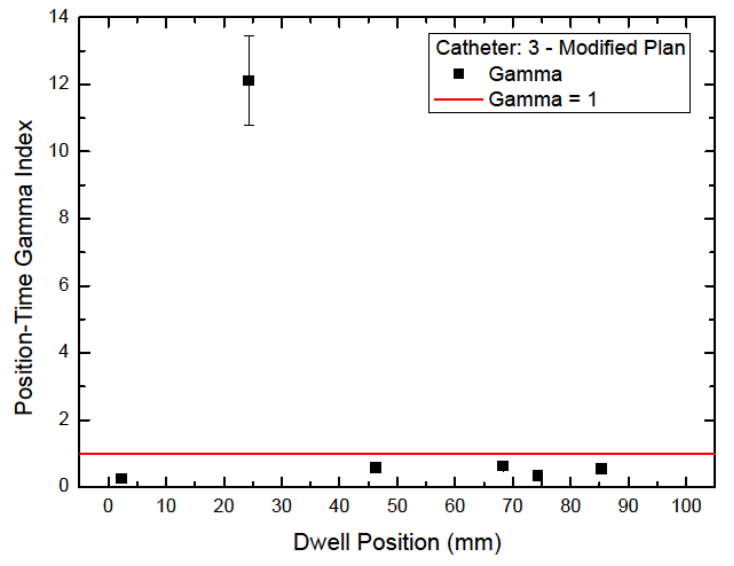

Figure 13 - Gamma analysis for Catheter 3 for the modified plan $(\mathrm{DTA}=1.3 \mathrm{~mm}, \mathrm{TTA}=0.3 \mathrm{~s})$. The introduced dwell position has a gamma value of $12.0 \pm 1.9$.

The addition of the new dwell position causes the gamma analysis of this catheter, seen in Figure 13, to fail the pass-fail criteria. A gamma value of $12.0 \pm 1.9$ (2 S.D) was calculated based on the agreement between the additional measured position and the nearest TPS planned dwell point (position $=24.3$, time $=11.3$ ). Comparing the position-time gamma index for the entire modified treatment to the original plan, Figure 14 indicates 11 modified dwell positions. Upon analyzing the modified treatment plan DICOM, these 11 alterations were verified to be the introduced changes.

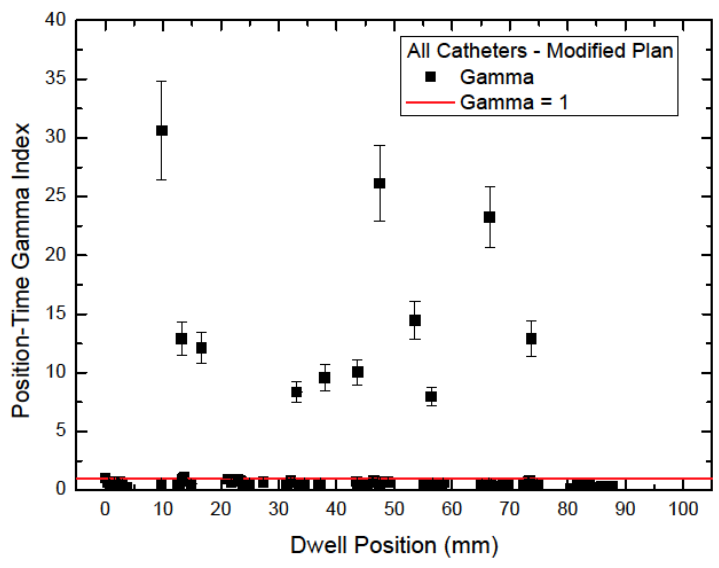

Figure 14 - Gamma analysis for all catheters for the modified plan $($ DTA $=1.3 \mathrm{~mm}$, TTA $=0.3 \mathrm{~s})$. Each gamma value larger than 1 represents a dwell position either removed or introduced into the plan. 


\section{E. Comparisons between "magic phantom" calculated dose, TPS and EBT3 film dose measurements}

The TPS DICOM file of the original plan was read by the MPh software, and the coordinates and value of each dose calculation were acquired. The software subsequently recalculated the dose at the same points using its own implementation of the TG-43U1 protocol, using the same planned parameters. Good agreement was found between the MPh software estimated doses for the 121 positions and the TPS, with values within $\pm 0.75 \%$ (2 S.D) suggesting that the dose calculation engine based on predetermined dwell positions and times is correct.

Two-dimensional 10x10 $\mathrm{cm}^{2}$ dose maps along the MPh plane were generated using the TG-43U1 calculation tool with $0.5 \times 0.5 \mathrm{~mm}^{2}$ pixel size. These were produced for the planned and measured source dwell positions and times of the original plan, and for the measured modified plan. The calculated 2D dose map for the measured dwell positions and times of the original plan is shown in Figure 15. The film was irradiated using the unmodified plan and processed to create a map with the same dimensions and resolution as the others.

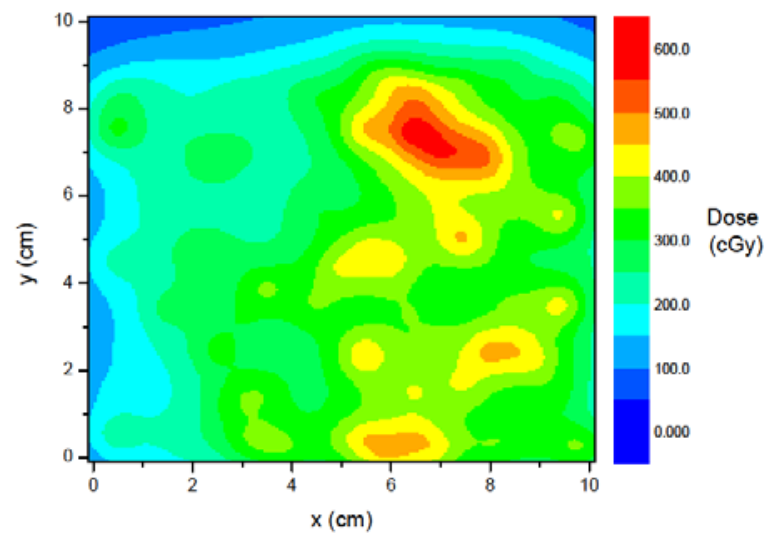

Figure 15 - Dose to the MPh detector plane calculated using TG-43U1, based upon measured dwell positions and times, without transit dose contribution.

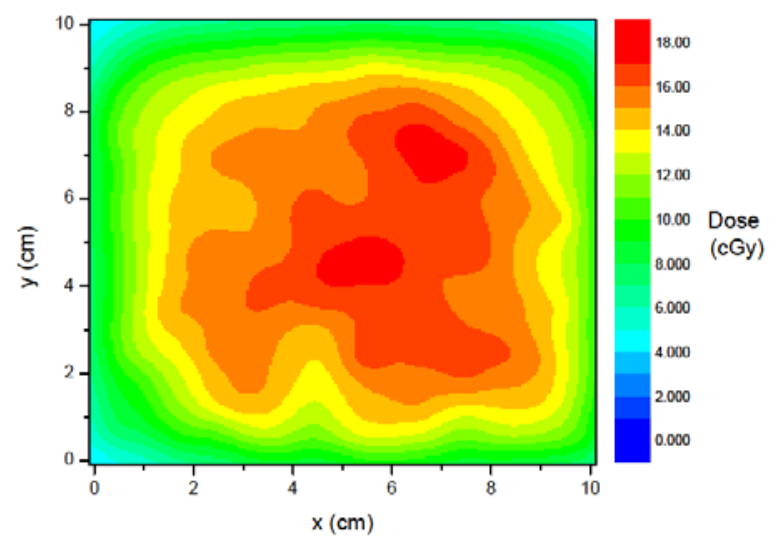

Figure 16 - The calculated total transit dose contribution, delivered to the MPh detector plane for the unmodified treatment plan.

The MPh system was also capable of estimating the contribution of the total transit dose for this plan by calculating the dose delivered for the sampled transit positions only. Seen in Figure 16, the total maximum transit dose was calculated to be $18 \mathrm{cGy}$ for the measured plan. This represents an average 
of $4.8 \pm 2.3 \%$ ( 2 S.D) increase in dose delivered when compared to the total dose delivered from the dwell positions only. This result is dependent on the source activity and for this particular plan the transit dose contribution could double when delivered directly after a source exchange, assuming the same transit times.

All dose distributions were compared using the 2D gamma analysis for varying dose difference and distance-to-agreement criteria, and the results are shown in Table 4. The dose difference value was calculated based upon the maximum dose within the compared maps. It is evident that by using a dose difference of $4 \%$ and distance-to-agreement of $3 \mathrm{~mm}$, a pass rate of over $95 \%$ could be achieved for both the MPh dose map without the transit dose and the film for the original plan. When considering the transit dose contribution, a pass rate of over $90 \%$ for all criteria examined was obtained. This suggests that the reduction of the dwell times is an appropriate method to compensate for the total transit dose when executing this plan. Using lower criteria values reduced the pass rate for film, most likely due to the registration of the film position to the MPh and the associated uncertainty in the film dose calculation.

Table 4 - Comparison of calculated dose maps against the reference TPS planned dwell positions and times.

\begin{tabular}{|c|c|c|c|}
\hline \multirow{2}{*}{\begin{tabular}{c}
\multirow{2}{*}{ Evaluated maps } \\
\cline { 2 - 4 }
\end{tabular}} & $2 \% / 2 \mathrm{~mm}$ & $3 \% / 3 \mathrm{~mm}$ & $4 \% / 3 \mathrm{~mm}$ \\
\cline { 2 - 4 } $\begin{array}{c}\text { Measured by the MPh - } \\
\text { original plan }\end{array}$ & 80.6 & 94.0 & 98.3 \\
\hline $\begin{array}{c}\text { Measured by the MPh - } \\
\text { original plan with } \\
\text { transit dose }\end{array}$ & 90.6 & 98.3 & 99.9 \\
\hline EBT3 film - original & & & \\
plan & 58.7 & & \\
\end{tabular}




\begin{tabular}{|c|c|c|c|}
\hline $\begin{array}{c}\text { Measured by MPh - } \\
\text { modified plan }\end{array}$ & 30.1 & 40.3 & 49.3 \\
& & & \\
\hline
\end{tabular}

The modified plan gave a pass rate of $49.3 \%$ for the dose difference of $4 \%$ and distance-to-agreement of $3 \mathrm{~mm}$, which was anticipated due to the simulated errors in afterloader delivery. Performing the dose calculations and using the $2 \mathrm{D}$ gamma analysis to compare the dose profiles allowed for additional verification of the treatment delivery, which could also be used to verify the dose calculations of the treatment planning system.

\section{Conclusion}

The novel "magic phantom" system and software has demonstrated its ability to verify treatment plans for HDR brachytherapy in terms of dwell position and times. Gross errors in source position and timing above $1.3 \mathrm{~mm}$ and $0.3 \mathrm{~s}$ have been shown to be measurable using the new position-time gamma index. The addition of a TG-43U1 calculation to the software allows for dosimetric information to be determined based upon the measured treatment plan and was shown to be in agreement with the TPS calculated values and with experimental EBT3 film results.

In future work, the "magic phantom" will be used to verify real patient plans and will translate measured dwell positions and time patterns to 3D dose calculations, including contributions of dose associated with source transition. The comparison of planned 3D doses by TPS and calculated values will complement the innovative position-time gamma analysis and provide comprehensive QA of the entire treatment delivery.

\section{Acknowledgements}

A. Espinoza would like to acknowledge the Rotary Club of Penrith Valley and Australian Rotary Health for their continued support and scholarship funding. Authors would like to acknowledge support from the Australian Research Council Grant No. DP 110104007. 
1 USNRC, "Loss of an iridium-192 source and therapy misadministration at Indiana Regional Cancer Center Indiana, Pennsylvania, on November 16, 1992", NUREG-1480, (U.S., Nuclear Regulatory Commission, Washington, DC, 1993).

2 J. Valentin, "ICRP 97 prevention of high-dose-rate brachytherapy accidents", Oxford, U.K., International Commission on Radiological Protection, Print (2005).

3 G. Kertzscher, A. Rosenfeld, S. Beddar, K. Tanderup, J.E. Cygler, "In vivo dosimetry: trends and prospects for brachytherapy”, Br J Radiol., 87(1041), 20140206 (2014)

4 M. J. Rivard, B. M. Coursey, L. A. DeWerd, W. F. Hanson, M. S. Huq, G. S. Ibbott, M. G. Mitch, R. Nath, J. F. Williamson, "Update of AAPM Task Group No. 43 Report: A revised AAPM protocol for brachytherapy dose calculations," Med. Phys. 31(3), 633-674 (2004).

5 B. R. Thomadsen, B. A. Erickson, P. J. Eifel, I. C. Hsu, R. R. Patel, D. G. Petereit, B. A. Fraass, M. J. Rivard, "A review of safety, quality management, and practice guidelines for high-dose-rate brachytherapy: Executive summary”, Pract. Radiat. Oncol., 4(2), 65-70 (2014).

6 H. Okamoto, A. Aikawa, A. Wakita, K. Yoshio, N. Murakami, S. Nakamura, M. Hamada, Y. Abe, J. Itami, "Dose error from deviation of dwell time and source position for high dose-rate 192Ir in remote afterloading system.”, J. Radiat. Res., 8, 1-8 (2014).

7 J. Duan, D. Macey, P. Pareek, I. Brezovich, "Real-time monitoring and verification of in-vivo high dose rate brachytherapy using a pinhole camera”, Med. Phys., 28(2), 167-173 (2001).

8 M. Batic, "A system for localization of high dose rate 192Ir source during brachytherapy treatment with silicon detectors”, 2009 IEEE Nuclear Science Symposium Conference Record, Orlando, FL (2009). 
9 T. Nakano, N. Suchowerska, M. M. Bilek, D. R. McKenzie, N. Ng, T. Kron, "High dose-rate brachytherapy source localization: positional resolution using a diamond detector", Phys. Med. Biol., 48, 2133-2146 (2003).

10 H. Song, J. Bowsher, S. Das, F. Yin, "Tracking brachytherapy sources using emission imaging with one flat panel detector", Med. Phys., 36(4), 1109-1111 (2009).

11 R. L. Smith, M. L. Taylor, L. N. McDermott, A. Haworth, J. L. Millar, R. D. Franich, "Source position verification and dosimetry in HDR brachytherapy using an EPID”, Med. Phys., 40(11), $111706(2013)$.

12 D. W. Rickey, D. Sasaki, J. Bews , "A quality assurance tool for high-dose-rate Brachytherapy", Med. Phys., 37(6), 2525-2532 (2010).

13 A. Manikandan, S. Biplab, P. A. David, R. Holla, T. R. Vivek, N. Sujatha, "Relative dosimetrical verification in high dose rate brachytherapy using two-dimensional detector array IMatriXX", J. Med. Phys, 36(3), 171-175 (2011).

14 A. Espinoza, B. Beeksma, M. Petasecca, I. Fuduli, C. Porumb, D. Cutajar, S. Corde, M. Jackson, M. L. Lerch, A. B. Rosenfeld, "The feasibility study and characterization of a two-dimensional diode array in "magic phantom" for high dose rate brachytherapy quality assurance", Med. Phys., 40(11), $111702(2013)$.

15 D. A. Low, W. B. Harms, S. Mutic, J. A Purdy, "A technique for the quantitative evaluation of dose distributions”, Med. Phys., 25(5), 656 (1998).

16 J. H. Wong, I. Fuduli, M. Carolan, M. L. Lerch, V. L. Perevertaylo, P. Metcalfe, A. B. Rosenfeld, "Characterization of a novel two dimensional diode array the "magic plate" as a radiation detector for radiation therapy treatment”, Med. Phys., 39(5), 2544-2558 (2012). 
17 A. H. Aldosari, A. Espinoza, D. Robinson, I. Fuduli, C. Porumb, S. Alshaikh, M. Carolan, M. L. F. Lerch, V. Perevertaylo, A. B. Rosenfeld, and M. Petasecca, "Characterization of an Innovative p-type Epitaxial Diode for Dosimetry in Modern External Beam Radiotherapy," IEEE Trans. Nucl. Sci., 60(6), 4705-4712 (2013).

18 J. A. Meli, A. S. Meigooni, R. Nath, "On the choice of phantom material for the dosimetry of 192Ir sources", Int. J. Radiat. Oncol., 14(3), 587-594 (1988).

19 J. F. Williamson, "Comparison of measured and calculated dose rates in water near I-125 and Ir192 seeds", Med. Phys., 18(4), 776 (1991).

20 A. C. Tedgren, G. A. Carlsson, "Influence of phantom material and dimensions on experimental ${ }^{192}$ Ir dosimetry", Med. Phys. 36(6), 2228 (2009).

21 I. Fudli, M. K. Newall, A. Espinoza, C. Porumb, M. Carolan, M. L. Lerch, P. Metcalfe, A. B. Rosenfeld, M. Pettasecca, "Multichannel Data Acquisition System comparison for Quality Assurance in external beam radiation therapy”, Rad. Meas. (In Press - June, 2014)

22 I. Fuduli, C. Porumb, A. Espinoza, A. H. Aldosari, M. Carolan, M. L. Lerch, P. Metcalfe, A. B. Rosenfeld, M. Petasecca, "A comparative analysis of multichannel Data Acquisition Systems for quality assurance in external beam radiation therapy”, J. Instrum., 9(06), T06003-T06003 (2014).

23 D. Granero, J. Pérez-Calatayud, E. Casal, F. Ballester, J. Venselaar, “A dosimetric study on the Ir192 high dose rate Flexisource”, Med. Phys., 33(12), 4578 (2006).

24 A. L. Palmer, A. Nisbet, D. A. Bradley, "Semi-3D dosimetry of high dose rate brachytherapy using a novel Gafchromic EBT3 film-array water phantom”, J. Phys.: Conf. Ser. 444, 012101 (2013).

25 T. A. D. Brown, K. R. Hogstrom, D. Alvarez, K. L. Matthews, K. Ham, J. P. Dugas, "Doseresponse curve of EBT, EBT2, and EBT3 radiochromic films to synchrotron-produced monochromatic x-ray beams", Med. Phys., 39(12), 7412-7 (2012). 
26 G. Massillon-JL, S. Chiu-Tsao, I. Domingo-Munoz, M. F. Chan, "Energy Dependence of the New Gafchromic EBT3 Film: Dose Response Curves for 50 KV, 6 and 15 MV X-Ray Beams”, Int. J. Med. Phys. Clin. Eng. Radiat. Oncol., 1(2), 60-65. (2012).

27 A. B. Rozenfeld, M. Zaider, "Method and apparatus for real time dosimetry", US patents, U.S. Patent No. 7,361,134, Issued: 22 April 2008; US Patent 7,972,259, Issued: 5 July 2011 